\title{
Evaluation of Low-Level Laser Therapy with Diode Laser for the Enhancement of the Orthodontic Tooth Movement: a Split-Mouth Study
}

\author{
Giovanni Matarese 1,", Marco Matarese ${ }^{2}$, Giacomo Picciolo ${ }^{3}$, Luca Fiorillo ${ }^{4}$ and Gaetano Isola ${ }^{5}$ \\ 1 Department of Biomedical, Odontostomatological Sciences and Morphological and Functional Images, \\ School of Dentistry, Via Consolare Valeria 1, University of Messina, 98125 Messina, Italy; \\ gmatarese@unime.it \\ 2 Department of Biomedical, Odontostomatological Sciences and Morphological and Functional Images, \\ School of Dentistry, Via Consolare Valeria 1, University of Messina, 98125 Messina, Italy; \\ matamarco94@gmail.com \\ 3 Department of Biomedical, Odontostomatological Sciences and Morphological and Functional Images, \\ School of Dentistry, Via Consolare Valeria 1, University of Messina, 98125 Messina, Italy; \\ giacomopicciolo94@gmail.com \\ 4 Department of Biomedical, Odontostomatological Sciences and Morphological and Functional Images, \\ School of Dentistry, Via Consolare Valeria 1, University of Messina, 98125 Messina, Italy; lucafiorillo@live.it \\ 5 Department of Biomedical, Odontostomatological Sciences and Morphological and Functional Images, \\ School of Dentistry, Via Consolare Valeria 1, University of Messina, 98125 Messina, Italy; \\ gaetanoisola@gmail.com \\ * Correspondence: gmatarese@unime.it; Tel.: +39-0902216904
}

\begin{abstract}
This study evaluated the effect of low-level laser irradiation induces by diode laser on the speed of orthodontic tooth movement of canines submitted to initial retraction. Twenty-four mandibular canines were retracted by using NiTi spring (force of $150 \mathrm{~g} /$ side). Thirteen of those were irradiated with a diode laser 980-nm diode laser (Wiser Laser Doctor Smile, Lambda) operating at an 810-nm wavelength ( $1 \mathrm{~W}$ of output power, continuous wave of $66.7 \mathrm{~J} / \mathrm{cm} 2)$ that was equipped with a $0.6-\mathrm{mm}$ optical fiber in continuous-wave mode. The canine retraction was accomplished by using prefabricated coil springs. The right of the mandible was chosen to be irradiated with the laser, whereas the left side was considered the control without laser irradiation. The laser was applied with 0-, 3-, 7-, and 14-day intervals. The amount of canine retraction was measured with a digital electronic caliper while the pain level was prompted by a patient questionnaire. The speed of tooth movement was significantly greater in the laser group than in the control group. The pain intensity was also at a lower level in the laser group. Our findings suggest that diode laser therapy can highly accelerate tooth movement during orthodontic treatment and can also effectively reduce pain level.
\end{abstract}

Keywords: Low level laser therapy, Diode laser, Orthodontic tooth movement, Pain, Split-mouth clinical trial

\section{Introduction}

The orthodontic movement is divided into three elements of tooth displacement: initial strain, lag phase, and progressive tooth movement. An initial strain of 0.4 to $0.9 \mathrm{~mm}$ occurs within about one week because of periodontal ligament displacement, bone strain, and extrusion [1,2]. Initial tooth displacement occurs within seconds, and this leads to a buildup of stresses within the periodontal ligament, which is supposed to be a critical rate-limiting step for the osteogenic response responsible for bone remodelling [3,4].

Overall, long-term treatment is one of the main reasons for patients to refrain from treatment $[5,6]$. It also has other disadvantages such as increasing the amount of decay, gingivitis and root 
resorption [7-9]. Thus, a non-invasive method of accelerating tooth movement physiologically is needed $[5,10]$. Low-level laser therapy (LLLT) is a therapy of laser with various photobiomodulation effects, and these potential effects of laser stimulate the velocity of tooth movement and thereby facilities the tooth movement in orthodontics [11,12].

LLLT, in the orthodontic field, has been demonstrated to be efficient in biomodulation, with inhibitory effects on analgesia and with stimulating action in tissue repair $[13,14]$. These stimulation effects are due to the capacity of LLL to accelerate metabolic changes and in bone tissue, to promote faster bone resorption and neoformation, both necessary for orthodontically induced tooth movement $[15,16]$.

Tissue-penetrating light can be generated by numerous types of currently available lasers, including He-Ne, Nd: YAG, and diode lasers, which are often used in the dental field. Surface absorption-type CO2 and Er: YAG laser light is also widely used. Diode laser light is highly penetrant and minimally absorbed by water $[17,18]$. In addition, diode lasers are inexpensive devices and easy to miniaturize, which has led to the development of a wide range of clinical applications. Laser therapy can aid in repairing the mucosa, controlling pain, and accelerating wound healing through low mechanical stress on the root surfaces and bactericidal and detoxifying effects [15,19].

At high power, diode lasers can be used to perform incision and hemostasis of soft tissue, whereas, at low power, they can be used for periodontal therapy $[20,21]$ and treatment of dentinal hypersensitivity [22].

With diode laser, even if the peak output is set high, it is possible to suppress the average output to a low level and to more efficiently apply the light energy to the tissue without creating thermal effects or thermal damage. Therefore, in the present study, we examined the application of a diode laser $(810 \mathrm{~nm})$ on the experimental clinical model of orthodontic tooth movement.

\section{Results}

All patients completed the study successfully. Table 1 shows the mean velocity of those movements in both diode laser and control groups. The speed of tooth movement was bigger in the diode laser side than in the control side (Figure 1).

\begin{tabular}{|c|c|c|c|}
\hline Group & Number of analyzed teeth & Mean speed of tooth movement & SD \\
\hline Diode laser & 12 & 2.122 & 0.223 \\
\hline Control group & 12 & 1.324 & 0.208 \\
\hline
\end{tabular}

Table 1: Speed of tooth movement in the analyzed canine of the mandible.

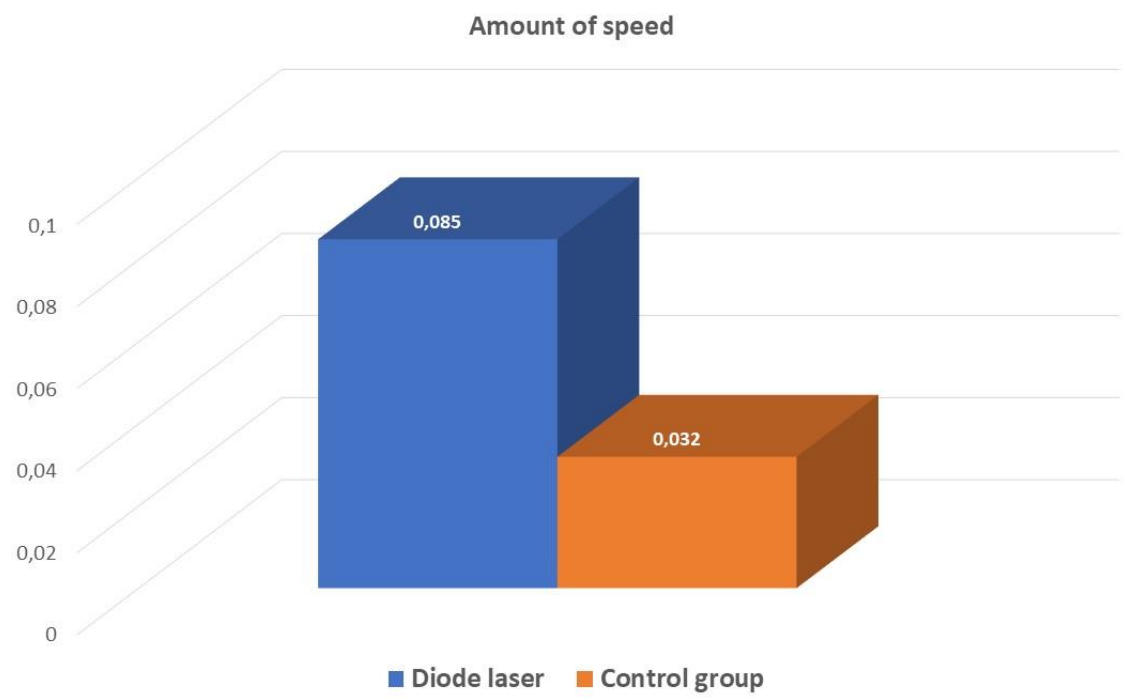


Figure 1: Amount of speed of tooth movement in diode laser and control group. The results are represented as median values.

Figure 2 shows the pain level during different treatment stages of diode laser and control groups, and the Table 4 shows mean rank values for the degree of pain during combined treatment stages. There was a significant difference in the degree of pain between the control and the diode laser side. The degree of pain was higher in the control side at each follow-up session (3, 7 and 14 days after laser activation) (Figure 4). After 6 months, none of the examined teeth presented damage developed in the adjacent periodontal ligament and dental tissues.

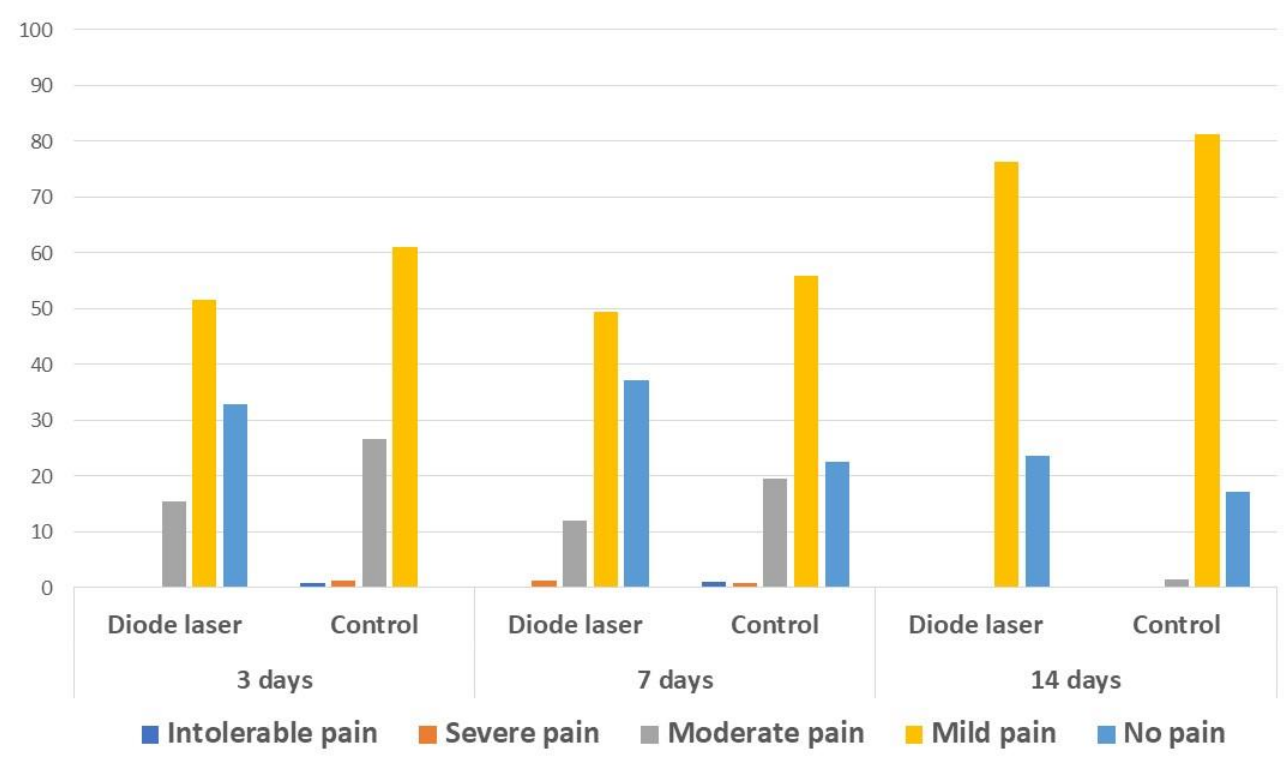

Figure 2: The results of the pain levels experienced in diode laser and control groups at each follow-up session (3, 7 and 14 days). The results are represented as a percentage.

\section{Discussion}

Few laboratory studies have evaluated the influence of laser on orthodontic movement in animals, but all of them proved that bone tissue that underwent LLL therapy showed an accelerated repair process, increasing bone neoformation and consequently increasing the speed of orthodontic tooth movement [2].

Orthodontic tooth movement involves both modeling and remodeling activities that are modulated by systemic factors such as nutrition, metabolic bone diseases, age, and drug usage history Biologically active substances, such as cytokines, interleukins (IL-1ß, IL-1RA), and enzymes, are expressed by cells within the periodontium in response to mechanical stress from orthodontic appliances [23-25].

According to several studies, LLLT is an effective tool used to prompt bone repair and modeling post-surgery [26,27]. This has referred to the biostimulation effect of the LLLT. This effect had been well studied in the medical field and proven to have an enhancement effect on fibroblast growth enhancement, wound healing, and bone repair [26].

This enhancement can be the result of osteoblasts proliferation and differentiation and intracellular changes in these cells [28-30]. Saito and Shimizu [31] studied the effects of low-power laser irradiation on bone regeneration in mid palatal suture during expansion in the rat and concluded that one-time or late irradiation (days 4-6) did not affect. However, irradiation during days 0-2 was the most effective. 
Biostimulation effects on the bone repair are directly dependent on the dose applied [32-33]. Different parameters have proven to be effective for several different lasers, inducing changes within cell cultures and leading to an increased healing effect, especially in patients with blood disorders [33,34]. Nevertheless, the optimal parameters have yet to be determined [35,36].

Luger et al. [37] used doses of about $64 \mathrm{~J} / \mathrm{cm} 2$ during 14 days, and although this dose could be excessive within the focused area, the authors believe that the scattering reduces the energy level of the laser beams to between 3 and $6 \%$ of its original intensity. In our study, the dose at each of the different points around the tooth was higher $(66.7 \mathrm{~J} / \mathrm{cm} 2)$ than the dose used by Luger et al. (64 $\mathrm{J} / \mathrm{cm} 2$ ), but the distribution of energy into six points surrounding the canine teeth could be more adequate due to a more homogeneous distribution of the energy.

Shimizu et al [38] showed that the orthodontic movement of laser-irradiated rats' teeth was $30 \%$ quicker than the nonirradiated rats due to the acceleration of bone formation as a result of the cellular stimulation promoted by LLLT. Our findings are similar to these reports. This ratio could be the biostimulation factor promoted by LLLT. Moreover, we observed that the velocity of tooth movement was bigger on the laser side than in the control side.

Harazaki et al. [39] showed that the low-level laser therapy is an effective tool to manage the post- adjustment orthodontic pain. Our findings in this research confirmed these previous findings; more specifically, the present study showed that during different treatment stages, the pain level of the diode laser group was less in amount than the control group.

In conclusion, the diode laser therapy used in this study was effective for the biostimulation of the periodontal apparatus during orthodontic treatment. The biostimulation determined, in the treated sites, an enhancement in the rate of tooth movement and the reduction of the tooth pain level due to the orthodontic movement.

Further studies are required to explain the mechanisms of laser biostimulation and clinical trials to optimize treatment parameters and discover other effects promoted by LLLT.

\section{Materials and Methods}

Fourteen patients of both genders (seven females and seven males), with a mean age of 13.1 years (range, 10.5-20.2 years) were selected for this study. The inclusion criteria were as follows: the need for extraction of first premolars due to biprotrusion or dental crowding; the presence of permanent dentition; the absence of any kind of systemic disease; no use any medication; and no previous orthodontic treatment. The local ethical committee of the University of Messina approved the study protocol (prot. 18/18 - 07/03/2018).

Twenty-four mandibular canines were evaluated, 12 lasers irradiated and 12 nonirradiated (control). Only one side of the arch was laser irradiated, representing the Diode Laser group, whereas the other side was not irradiated and then was considered the Control Group. The initial retraction of the canines started after 3 months of the premolar extractions, and it was further evaluated for 4 months. Metallic brackets, 0.022-0.028-inch slot (Ormco Corp., Orange, CA), were placed on canines, whereas the premolars and molars were properly banded. Bilaterally segmented arches made of stainless steel, 0.016 , were adopted in conjunction with a closed $\mathrm{Ni}$-Ti coil spring ( $9 \mathrm{~mm}$ in length, G\&H, Franklin, IN, USA), delivering a force of $50 \mathrm{~g} /$ side at maximum extension for the initial retraction of the canines (Figure $3 \mathrm{a}$ and $\mathrm{b}$ ). This force was measured with a dynamometer (commercial brand, Morelli).

Spring reactivation was made every 21 days with the same force value $(150 \mathrm{~g})$ and repeated until the closing of the extraction space. The amount of tooth movement was measured in millimeters was prompted by measuring the distance between the tip of the mesial cusp of the first molar and the tip of the canine cusp.

The speed of the tooth movement was obtained from the following formula $V=d / t$ in which $V$ was the speed of the canine movement, $\mathrm{d}$ is the amount of canine movement in millimeters at the end of treatment, and $t$ the time useful to accomplish the movement.

The measurement was done by using a digital caliper (Mitutoyo, Japan) before initiating the orthodontic treatment and recorded. At each reactivation interval, new impressions for each patient 
of both upper and lower jaws were taken. Then a new measurement of the previous distance was recorded. On every reactivation date, the patient was asked about the pain experienced during the bygone period. These responses were ranked according to a visual pain scale and were also organized in a schedule.

The right side of the mandible was chosen to be irradiated with the laser beam, whereas the left side was considered the control without irradiation.

The treatment was performed with a 980-nm diode laser (Wiser Laser Doctor Smile, Lambda) operating at an 810-nm wavelength ( $1 \mathrm{~W}$ of output power, continuous wave of $66.7 \mathrm{~J} / \mathrm{cm} 2)$ that was equipped with a $0.6-\mathrm{mm}$ optical fiber in continuous-wave mode. The areas were chosen to be irradiated the lingual and buccal periodontal ligament of the canines. Tree areas Cervical, Middle and Apical, were irradiated. The cervical area was equipped with a diode laser for $10 \mathrm{~s}$. The middle area was equipped with a diode laser for $20 \mathrm{~s}$. The apical area was equipped with a diode laser for 10 s. The total energy density (dose) at each application was $8 \mathrm{~J}(2 \times 40 \mathrm{~s} \times 100 \mathrm{~mW})$. Protective eyewear was worn by the patients and the clinician to prevent injury from laser wavelength exposure. The laser regimen was performed after the closed NiTi coil spring was installed or activated (day 0), and on, 3-, 7-, and 14-day intervals after every activation.

At every 21-day interval, the distance measurement was compared between the diode laser and control group. After 6 months, the diode laser and control groups were examined by periapical radiographs to see if any damage developed in the adjacent periodontal ligament and dental tissues.

Every patient was asked about the pain experienced after spring activation describing a single value of no pain (0), mild pain (1), moderate pain (2), severe pain (3), intolerable pain (4).

\subsection{Statistical Analysis}

Numeric data are expressed as the mean and standard deviation (SD), and categoric variables are expressed as numbers and percentages. The data were compared by two-sample t-tests. To analyze the differences in pain levels between the diode laser and control groups during combined treatment stages, Man-Whitney U test was done. Statistical analyses were performed using SPSS software, version 11.0, for Windows. A P value <.05 was considered statistically significant.

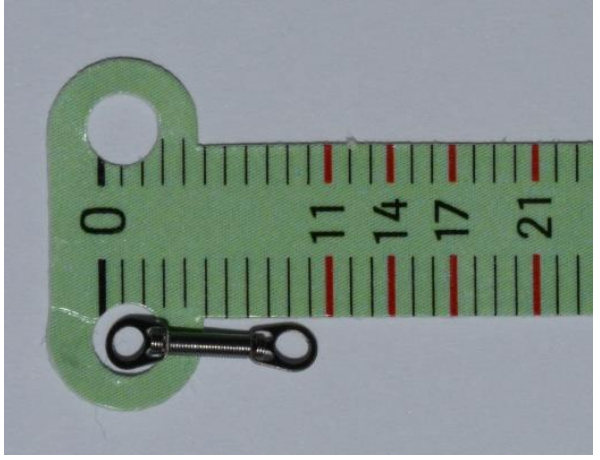

(a)

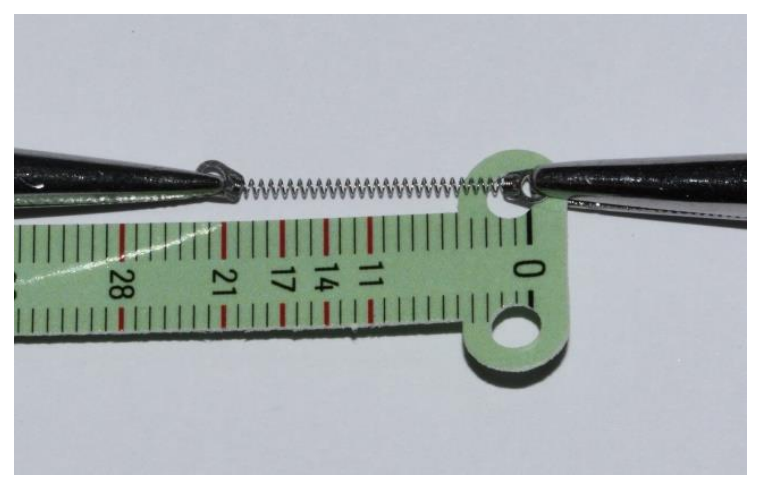

(b)

Figure 3: (a) The closed NiTi coil spring of $9 \mathrm{~mm}$ used for the study; (b) The NiTi coil spring activated with a force of $50 \mathrm{~g}$

\section{Acknowledgement}

This work has been performed with funding by the Department of Biomedical, Odontostomatological Sciences and Morphological and Functional Images, University of Messina, Messina, Italy. The authors declare that they have no conflict of interest in the present study.

\section{References}

1. Verna, C.; Cattaneo, P.M.; Dalstra M. Corticotomy affects both the modus and magnitude of orthodontic tooth movement. Eur. J. Orthod. 2018, 40, 107-112. 
2. Isola, G.; Matarese, G.; Cordasco, G.; Perillo, L.; Ramaglia, L. Mechanobiology of the tooth movement during the orthodontic treatment: a literature review. Minerva Stomatol. 2016, 65, 299-327.

3. Lucchese, A.; Matarese, G; Ghislanzoni, L.H.; Gastaldi, G; Manuelli, M.; Gherlone, E. Efficacy and effects of palifermin for the treatment of oral mucositis in patients affected by acute lymphoblastic leukemia. Leuk. Lymphoma 2016, 57, 820-7.

4. Antonarakis, G.S.; Joss, C.U.; Triaca, A.; Kuijpers-Jagtman, A.M.; Kiliaridis, S. Gingival recessions of lower incisors after proclination by orthodontics alone or in combination with anterior mandibular alveolar process distraction osteogenesis. Clin. Oral Investig. 2017, 21, 2569-2579.

5. Bock, N.; Ruehl, J; Ruf, S. Orthodontic Class II:1 treatment-efficiency and outcome quality of Herbst-multibracket appliance therapy. Clin. Oral Investig. 2018, 22, 2005-2011.

6. Lucchese, A.; Matarese, G.; Manuelli, M.; Ciuffreda, C.; Bassani, L.; Isola, G; Cordasco, G.; Gherlone, E. Reliability and efficacy of palifermin in prevention and management of oral mucositis in patients with acute lymphoblastic leukemia: a randomized, double-blind controlled clinical trial. Minerva Stomatol. 2016; 65, 43-50.

7. Pereira, S.; Nogueira, L.; Canova, F.; Lopez, M.; Silva, H.C. IRAK1 variant is protective for orthodontic-induced external apical root resorption. Oral Dis. 2016, 22, 658-664.

8. Matarese, G; Isola, G; Ramaglia, L; Dalessandri, D; Lucchese, A; Alibrandi, A; Fabiano, F.; Cordasco, G. Periodontal biotype: characteristic, prevalence and dimensions related to dental malocclusion. Minerva Stomatol. 2016, 65, 231-238.

9. Matarese, G.; Isola, G., Anastasi, G.P.; Cutroneo, G.; Favaloro, A.; Vita, G.; Cordasco, G.; Milardi, D.; Zizzari, V.L.; Tetè, S; Perillo, L. Transforming Growth Factor Beta 1 and Vascular Endothelial Growth Factor levels in the pathogenesis of periodontal disease. Eur. J. Inflamm. 2013, 11, 479-488.

10. Piancino, M.G.; Isola, G.; Cannavale, R.; Cutroneo, G; Vermiglio, G; Bracco, P; Anastasi, G.P. From periodontal mechanoreceptors to chewing motor control: A systematic review. Arch. Oral Biol. 2017, 78, 109-121.

11. Yu, C.C.; Chen, Y.R.; Lin, J.C. Line Laser as an Assistance for Facial and Dental Midlines Evaluation in Single-Splint Orthognathic Surgery. J. Craniofac. Surg. 2017, 28, 1786-1788.

12. Isola, G.; Matarese, G.; Lo Giudice, G.; Briguglio, F.; Alibrandi, A.; Crupi, A.; Cordasco, G.; Ramaglia, L. A New Approach for the Treatment of Lateral Periodontal Cysts with an 810-nm Diode Laser. Int. J. Periodontics Restorative Dent. 2017, 37, e120-e129.

13. Reichelt, J.; Winter, J.; Meister, J.; Frentzen, M.; Kraus, D. A novel blue light laser system for surgical applications in dentistry: evaluation of specific laser-tissue interactions in monolayer cultures. Clin. Oral Investig. 2017, 21, 985-994.

14. Isola, G.; Matarese, G.; Cervino, G.; Matarese, M., Ramaglia. L; Cicciù, M. Clinical Efficacy and Patient Perceptions of Pyogenic Granuloma Excision Using Diode Laser Versus Conventional Surgical Techniques. J. Craniofac. Surg. 2018, Jul 13. doi: 10.1097/SCS.0000000000004734.

15. Kanaguchi Arita, A.; Yonemitsu, I; Ikeda, Y.; Miyazaki, M; Ono, T. Low-intensity pulsed ultrasound stimulation for mandibular condyle osteoarthritis lesions in rats. Oral Dis. 2018, 24, 600-610.

16. Lucchese, A.; Manuelli, M.; Bassani, L.; Albertini, P.; Matarese, G.; Perillo, L.; Gastaldi, G.; Gherlone, E.F. Fiber reinforced composites orthodontic retainers. Minerva Stomatol. 2015, 64, 323-333.

17. Mohajerani, S.H.; Tabeie, F.; Bemanali, M.; Tabrizi, R. Effect of Low-Level Laser and Light-Emitting Diode on Inferior Alveolar Nerve Recovery After Sagittal Split Osteotomy of the Mandible: A Randomized Clinical Trial Study. J. Craniofac. Surg. 2017, 28, e408-e411.

18. Matarese, G.; Ramaglia, L.; Cicciù, M.; Cordasco, G.; Isola, G. The Effects of Diode Laser Therapy as an Adjunct to Scaling and Root Planing in the Treatment of Aggressive Periodontitis: A 1-Year Randomized Controlled Clinical Trial. Photomed. Laser Surg. 2017, 35, 702-709.

19. Isola, G.; Cicciù, M.; Fiorillo, L.; Matarese, G. Association Between Odontoma and Impacted Teeth. J. Craniofac. Surg. 2017, 28, 755-758.

20. Roncati, M.; Gariffo, A. Three Years of a Nonsurgical Periodontal Treatment Protocol to Observe Clinical Outcomes in $\geq 6$-mm Pockets: A Retrospective Case Series. Int. J. Periodontics Restorative Dent. 2016, 36, 189-197.

21. Isola, G.; Matarese, G.; Williams, R.C.; Iorio-Siciliano, V.; Alibrandi, A.; Cordasco, G.; Ramaglia, L. The effects of a desiccant agent in the treatment of chronic periodontitis: a randomized, controlled clinical trial. Clin. Oral Investig. 2018, 22, 791-800. 
22. Galafassi, D.; Scatena, C.; Galo, R.; Curylofo-Zotti, F.A.; Corona, S.A.M.; Borsatto, M.C. Clinical evaluation of composite restorations in Er:YAG laser-prepared cavities re-wetting with chlorhexidine. Clin. Oral Investig. 2017, 21, 1231-1241.

23. Spadari, G.S.; Zaniboni, E.; Vedovello, S.A.; Santamaria, M.P.; do Amaral, M.E.; Dos Santos, G.M.; Esquisatto, M.A.; Mendonca, F.A.; Santamaria, M. Jr. Electrical stimulation enhances tissue reorganization during orthodontic tooth movement in rats. Clin. Oral Investig. 2017, 21, 111-120.

24. Lo Giudice, G.; Lo Giudice, R.; Matarese, G.; Isola, G.; Cicciù, M.; Terranova, A.; Palaia, G.; Romeo, U. Evaluation of magnification systems in restorative dentistry. An in-vitro study. Valutazione dei sistemi di ingrandimento in odontoiatria conservativa e restaurativa. Studio in vitro. Dental Cadmos 2015, 83, 296-305.

25. Isola, G.; Anastasi, G.P.; Matarese, G.; Williams, R.C.; Cutroneo, G.; Bracco, P.; Piancino, M.G. Functional and molecular outcomes of the human masticatory muscles. Oral Dis. 2017 Nov 20. doi: 10.1111/odi.12806.

26. Fischer, K.R.; Mühlemann, S.; Jung, R.E.; Friedmann, A.; Fickl, S. Dimensional Evaluation of Different Ridge Preservation Techniques with a Bovine Xenograft: A Randomized Controlled Clinical Trial. Int. J. Periodontics Restorative Dent. 2018, 38, 549-556.

27. Martuscelli, R.; Toti, P.; Sbordone, L.; Guidetti, F.; Ramaglia, L.; Sbordone, C. Five-year outcome of bone remodelling around implants in the maxillary sinus: assessment of differences between implants placed in autogenous inlay bone blocks and in ungrafted maxilla. Int. J. Oral Maxillofac. Surg. 2014, 43, 1117-26.

28. Lo Giudice, G.; Lizio, A.; Lo Giudice, R.; Centofanti, A.; Rizzo, G.; Runci, M.; Alibrandi, A.; Cicciù, M. The Effect of Different Cleaning Protocols on Post Space: A SEM Study. Int. J. Dent. 2016, 2016, 1907124.

29. Fujioka-Kobayashi, M.; Schaller, B.; Zhang, Y.; Kandalam, U.; Hernandez, M.; Miron, R.J. Recombinant human bone morphogenetic protein (rhBMP)9 induces osteoblast differentiation when combined with demineralized freeze-dried bone allografts (DFDBAs) or biphasic calcium phosphate (BCP). Clin. Oral Investig. 2017, 21, 1883-1893.

30. Lo Giudice, G.; Matarese, G.; Lizio, A.; Lo Giudice, R.; Tumedei, M.; Zizzari, V.L.; Tetè S. Invasive Cervical Resorption: A Case Series with 3-Year Follow-Up. Int. J. Periodontics Restorative Dent. 2016, 36, 103-109.

31. Saito, S.; Shimizu, N. Stimulatory effects of low-power laser irradiation on bone regeneration in midpalatal suture during expansion in the rat. Am. J. Orthod. Dentofacial Orthop. 1997, 111, 525-532.

32. Cicciù, M.; Herford, A.S.; Cervino, G.; Troiano, G.; Lauritano, F.; Laino, L. Tissue Fluorescence Imaging (VELscope) for Quick Non-Invasive Diagnosis in Oral Pathology. J. Craniofac. Surg. 2017, 28, e112-e115.

33. Tao, X.; Yao, J.W.; Wang, H.L.; Huang, C. Comparison of Gingival Troughing by Laser and Retraction Cord. Int. J. Periodontics Restorative Dent. 2018, 38, 527-532.

34. Isola, G.; Matarese, G.; Cordasco, G.; Rotondo, F.; Crupi, A.; Ramaglia, L. Anticoagulant therapy in patients undergoing dental interventions: a critical review of the literature and current perspectives. Minerva Stomatol. 2015, 64, 21-46.

35. Lin, J.C.; Nevins, M.; Kim, D.M. Laser De-epithelialization of Autogenous Gingival Graft for Root Coverage and Soft Tissue Augmentation Procedures. Int. J. Periodontics Restorative Dent. 2018, 38, 405-411.

36. Cannavale, R.; Matarese, G.; Isola, G.; Grassia, V.; Perillo, L. Early treatment of an ectopic premolar to prevent molar-premolar transposition. Am. J. Orthod. Dentofacial Orthop. 2013, 143, 559-569.

37. Luger, E.J.; Rochkind, S.; Wollman, Y.; Kogan, G.; Dekel, S. Effect of low-power laser irradiation on the mechanical properties of bone fracture healing in rats. Lasers Surg. Med. 1998, 22, 97-102.

38. Shimizu, N.; Yamaguchi, M.; Goseki, T.; Shibata, Y.; Takiguchi, H.; Iwasawa, T.; Abiko, Y. Inhibition of prostaglandin E2 and interleukin 1-beta production by low-power laser irradiation in stretched human periodontal ligament cells. J. Dent. Res. 1995; 74, 1382-1388.

39. Harazaki, M.; Takahashi, H.; Ito, A.; Isshiki, Y. Soft laser irradiation induced pain reduction in orthodontic treatment. Bull. Tokyo Dent. Coll. 1998, 39, 95-101. 